\title{
« Rosebud »: le motif du secret dans la fiction biographique chez Welles et Davies
}

Julie Wolkenstein

\section{OpenEdition}

\section{Journals}

Édition électronique

URL : http://journals.openedition.org/recherchestravaux/138

DOI : 10.4000/recherchestravaux.138

ISSN : 1969-6434

Éditeur

UGA Éditions/Université Grenoble Alpes

Édition imprimée

Date de publication : 15 avril 2006

Pagination : 109-119

ISBN : 2-9518254-8-X

ISSN : 0151-1874

Référence électronique

Julie Wolkenstein, « «Rosebud » : le motif du secret dans la fiction biographique chez Welles et

Davies », Recherches \& Travaux [En ligne], 68 | 2006, mis en ligne le 06 novembre 2008, consulté le 10 décembre 2020. URL : http://journals.openedition.org/recherchestravaux/138 ; DOI : https://doi.org/ 10.4000/recherchestravaux.138 
Julie WOLKENSTEIN

Université de Caen

\section{«Rosebud» : le motif du secret dans la fiction biographique chez Welles et Davies}

Les deux œuvres associées ici ne bénéficient pas d'une notoriété équivalente: le roman de l'auteur canadien anglophone Robertson Davies est beaucoup moins célèbre, en France du moins, que Citizen Kane; je vais donc tenter d'en résumer les enjeux, en les rapprochant du film de Welles ${ }^{1}$. Il existe certaines similitudes entre les biographies respectives de Welles et de Davies: ils appartiennent à la même génération (le premier est né en 1915, le second en 1913) et grandissent à l'écart des grandes métropoles, l'Ontario pour Davies, le Wisconsin pour Welles. La carrière de Davies présente une variété, une mobilité qui rappelle celle de Welles: comme lui, il est acteur à la fin des années 1930, puis écrit pour la scène, avant de devenir chroniqueur, critique, essayiste, rédacteur en chef d'une revue canadienne, professeur de littérature anglaise à Toronto, et enfin romancier dans les années 1970. Ce sont tous deux des caméléons, dont les multiples facettes (de son côté Welles s'est illustré non seulement comme cinéaste, mais aussi comme scénariste, comédien, directeur de troupe, et s'est fait connaître par une émission de radio) se retrouvent dans les personnages dont ils inventent la vie et interrogent l'identité, en suggérant qu'une clef unique la résume et l'élucide.

1. Ce dernier a sans doute exercé une influence sur son écriture, comme le confirme la lecture d'une autre fiction de Davies, cinéphile averti, Fantômes et Cie, qui dresse la généalogie d'un personnage par l'intermédiaire d'une projection publique de séquences cinématographiques inspirées du cinéma soviétique de l'entre-deux guerres, soit contemporaines de Welles et pratiquant des innovations formelles comparables, notamment sur le plan du montage. 
Le titre français du roman de Davies, Un homme remarquable, publié en 1985, ne tient aucun compte de l'original, What's bred in the bone, c'est-àdire littéralement "ce qui a été mis dans la moelle", lui-même traduction médiévale d'un proverbe latin qui dit ceci: «Ce qui a été mis dans la moelle ne sort plus de la chair». Cette citation tronquée propose au lecteur de découvrir les ressorts secrets et déterminants d'une personnalité, celle de Francis Cornish, figure fictive dont le roman retrace la biographie.

Les parentés entre ces biographies fictives, celle de Cornish et celle de Kane sont nombreuses: tout d'abord, il s'agit dans les deux cas d'inventer une vie singulière, mais chargée d'incarner plus généralement le destin d'une civilisation récente, de dépasser le portrait d'un Américain pour dessiner une histoire collective, celle du Nouveau Monde dans la première moitié du $\mathrm{XX}^{\mathrm{e}}$ siècle.

Toutes deux se présentent comme une enquête, une investigation, recourent au genre biographique sous une forme problématique, donnant à voir, non le résultat du travail du biographe, mais les difficultés qu'il rencontre dans la mise en œuvre des sources, les limites et les contradictions de son travail préparatoire, ses déficiences, son impuissance.

Enfin, elles jouent avec l'hypothèse - illusoire? - d'un principe secret, d'une énigme, d'un motif souterrain qui permettrait de saisir l'essence du personnage, et, ce faisant, réfléchissent aussi au statut de la fiction, à sa possible réduction à une interprétation univoque.

Le statut du personnage, individu ou type, figure particulière ou incarnation d'un destin national, se complique chez Welles dans la mesure où Charles Foster Kane peut être - et l'a d'ailleurs été - identifié à un homme réel: William Randolph Hearst. Au plus fort de la polémique qui suit la réalisation de Citizen Kane, l'auteur exprime ainsi cette ambiguïté: "Ce film n'emprunte rien à la biographie de M. Hearst ni à la vie de qui que ce soit d'autre. D'un autre côté, s'il n'y avait pas eu M. Hearst et un certain nombre de barons de la finance, jamais Kane n'aurait existé ${ }^{2}$ ». Dans la monographie qu'il consacre à Citizen Kane, Dominique Château commente ces propos en soulignant que «le film était censé renvoyer à la réalité davantage par son discours sur un type que sur un individu ${ }^{3}$ » et il ajoute aussi :

2. Cité par Barbara Leaming, Orson Welles, New York, Viking, 1985; trad. par JeanPierre Carasso et Bruno Monthureux, Éditions Mazarine, «Biographie», 1986, p. 224.

3. D. Château, Citizen Kane, L'Interdisciplinaire, 1993, p. 24. 
le récit des conquêtes et des frasques du magnat ne veut pas se limiter au portrait d'une seule personne, à la pure et simple exactitude d'une biographie fidèle. [...] Welles amalgame les références à William Randolph avec des traits extrinsèques qui procèdent souvent du renvoi à d'autres figures - par exemple, Susan Alexander rappelle Marion Davies, la fiancée de Hearst, mais, d'une part, celle-ci était réputée talentueuse et, d'autre part, c'est un autre milliardaire qui fit construire l'opéra de Chicago pour un motif comparable. Par-delà l'anecdote donc, le personnage façonné par le cinéaste représente un rôle social caractéristique de l'époque [et] atteint incontestablement ce degré de généralité. Au moment où la réalisation du film commence, ce profil est en voie de disparition; au pouvoir personnel [...] succède la loi impersonnelle des corporations bancaires et des holdings qui travaillent sous le couvert de l'anonymat ${ }^{4}$.

La vie de Francis Cornish, le héros de What's bred in the bone, peut aussi se lire de manière extensive comme une réflexion sur l'identité canadienne dans la même période, et sa biographie converge en plusieurs points avec celle de Kane: les deux personnages sont des immigrés de la seconde génération au moins; les origines de leur famille, installée dans une communauté rurale reculée, dans des conditions matérielles et climatiques difficiles, sont modestes; l'accès spectaculaire de leurs ascendants à la fortune est dû à des facteurs typiquement territoriaux: le commerce du bois pour Cornish, et, plus mythique, la mine d'or pour Kane; tous deux sont donc les premiers héritiers des pionniers, disposant de moyens considérables, mais que leurs goûts personnels détourneront de la finance et porteront vers des carrières plus créatives ou du moins récréatives (la peinture pour Cornish, le journalisme pour Kane). Produits du rêve américain devenu réalité, ils sont aussi représentatifs des relations du Nouveau Monde avec la culture et le patrimoine européens: les alliances que concluent Kane, par son premier mariage avec la nièce du Président des États-unis, séduite à l'occasion d'un séjour à Paris, et la mère de Cornish avec un officier britannique, rencontré lors d'un bal à Buckingham Palace, révèlent la nécessité fantasmatique d'un lien avec l'aristocratie de l'Est pour devenir un héros américain. Tous deux consacrent une grande partie de leur fortune à la collection d'œuvres d'art importées de l'Ancien Continent et s'inscrivent dans la lignée du personnage d'Adam Verver, dans La Coupe d'or de Henry James, accumulant des acquisitions dont la valeur n'est pas toujours reconnue par leurs compatriotes (à propos d'une statue de Vénus achetée par Kane, un observateur s'étonne qu'on puisse payer "si cher pour une femme sans tête»). Leur besoin de saturer, sinon leur pays, du moins leur espace personnel de spécimens étrangers est inscrit dans une perspective internationale stéréotypée: l'Amérique

4. Ibid., p. 20. 
rachète ainsi la jeunesse de sa culture; «L'Europe produit des chefs-d'œuvre depuis deux mille ans et je n'en achète que depuis cinq ans», déclare Kane. Sa puissance financière lui assure aussi l'asservissement des Européens - les deux seuls personnages qui viennent d'Europe sont le maitre de chant de Susan et le majordome. Chez Davies, c'est un Italien qui explique cette volonté de s'approprier, sinon les Européens (et encore, la famille de Cornish achète-telle un beau parti anglais pour sa mère), du moins leurs objets, par l'effondrement et la nostalgie de la spiritualité des hommes de la Renaissance: «La passion moderne pour les œuvres d'art du passé fait partie de ce terrible désir de certitude. [...] Pourquoi de riches Américains paient-ils des sommes fabuleuses pour des tableaux de maitres anciens qu'ils comprennent ou non, qu'ils aiment ou non, si ce n'est pour importer dans leur pays la certitude dont je vous parle? Leur vie publique est un cirque, mais la National Gallery de Washington recèle peut-être une parcelle du divin, une parcelle du confort de la splendeur divine ${ }^{5}$ ».

La collection est chaque fois présentée comme pléthorique, démesurée: Cornish achève son existence "dans un lieu qui ressemblait à la réserve d'un antiquaire à laquelle il ajoutait sans cesse le contenu d'autres caisses, cartons et paquets ${ }^{6} \%$. On songe aux derniers plans de Citizen Kane: les innombrables caisses empilées dans le hall de Xanadu, filmées comme des gratte-ciel, qui disent à la fois le gigantisme propre à l'urbanisme américain et la construction de cette nation sur un butin artistique d'origine européenne. Le mécénat est le corollaire de leur passion à tous deux, mais toujours entaché d'escroquerie: Kane fait construire un opéra parce que c'est le seul moyen pour que sa seconde femme, Susan Alexander, totalement dépourvue de talent, se produise comme cantatrice, dans une adaptation de Salammbô; Cornish, pris à son propre piège, renonce de justesse à financer l'acquisition par le musée d'Ottawa d'un tableau attribué à un maitre allemand du gothique tardif, mais dont il est en fait l'auteur.

La biographie fictive est donc bien, avec des traits communs, l'instrument d'une représentation plus large: une nation et une culture émergentes se dotent, comme dans l'épopée, d'une figure symbolique, à cette différence près, essentielle, que l'épopée américaine de Welles ou de Davies, tournée vers l'Est, inverse et pervertit les valeurs héroïques du genre antique. La spécificité géographique, historique et culturelle de leurs pays se reflète exactement dans la trajectoire personnelle des héros: Kane proclame à plusieurs

5. R. Davies, Un homme remarquable, trad. par Lisa Rosenbaum, Points/Seuil, 1992, p. 400 .

6. Ibid., p. 492. 
reprises qu'il se définit avant tout et exclusivement comme "un Américain" et Davies superpose au portrait de Cornish une allégorie du Canada, «un pays introverti qui essaie désespérément de se comporter comme un extraverti ${ }^{7}$ ». Ou encore:

le Canada, ce garçon de ferme aux grands yeux innocents, était en train de devenir un citadin déluré. [...] le petit pays au grand corps qui avait toujours été introverti - introversion qui s'était manifestée par le préjugé loyaliste, c'est-àdire le refus de se faire libérer par la force militaire de son puissant voisin de ce que ce dernier jugeait être un intolérable joug colonial - s'efforçait maintenant d'adopter l'extraversion dudit voisin. Parce que le Canada ne pouvait pas vraiment comprendre l'extraversion américaine, il en imitait les éléments les plus voyants, et le résultat était souvent d'un goût douteux ${ }^{8}$.

Le fait que ces héros américains soient un faussaire (Cornish) et un falsificateur (Kane) n'a pas pour seule conséquence la représentation critique d'une nation culturellement condamnée au plagiat (à leur époque du moins), mais complique aussi la tâche de leurs biographes et la réflexion sur vérité et mensonge qu'engage le recours à la forme de la fiction biographique.

La biographie, comme la fiction, et surtout la fiction moderne, met en jeu, en relation et quelquefois en opposition, deux champs d'une expérience humaine, la vie privée et la vie publique. Les frontières de l'intime reculent et la surface sociale projette avec plus ou moins d'authenticité les pensées et désirs profonds du sujet. Dans les deux œuvres qui nous intéressent, cette tension est fondamentale. Kane et Cornish sont impliqués dans les destins politiques de leur pays: Kane manipule l'opinion dans ses journaux, brigue un poste de gouverneur, rêve de la Maison Blanche. Homme public, il voit sa carrière brisée par la révélation médiatique de son adultère. "Sa vie privée appartenait au public", commente un journaliste. Welles n'anticipe pas seulement, pour s'en défendre, les accusations qu'on fera à son propre film, mais aussi les risques de l'exhibition de soi, ses revers, dans un système où la démocratie doit composer avec une nécessité d'absolue transparence, entretenue par les médias. L'enjeu dramatique de Citizen Kane repose d'ailleurs significativement sur l'élucidation d'un secret, ultime noyau qui résiste au harcèlement intime des observateurs, mais pas au regard du spectateur, pas vraiment un noyau, mais presque: un bouton de rose, «Rosebud». Chez Davies, le héros introverti œuvre aussi pour son pays, mais dans l'ombre: comme son père, il est agent secret au service de l'Angleterre et cette version obscure de l'activité politique trouve un écho dans la dissimulation, les mensonges, la 
clandestinité qui entourent sa vie privée. Lui aussi exhibe son intimité, mais de façon travestie: le tableau allégorique qu'il manque céder au musée d'Ottawa, intitulé Les Noces de Cana, réserve au seul lecteur le privilège d'une interprétation fiable.

La réflexion sur les domaines privé et public, amplifiée par les fonctions respectives de Kane et de Cornish, rencontre dans les deux cas l'écart entre vérité et mensonge: le premier, manipulateur d'opinion, falsificateur - tous les journaux qu'il possède célèbrent unanimement le talent de sa femme Susan - est aussi obsédé par la recherche de la vérité (en témoignent : la profession de foi qu'il publie lorsqu'il reprend, à ses débuts, le New York Inquirer; le choix de laisser éclater le scandale qui compromet définitivement sa carrière politique en révélant sa liaison; et surtout la critique qu'il publie lors de la première de Salammbô: commencée par son meilleur ami Leland comme un éreintement, elle est achevée "dans le même esprit " par Kane luimême, et paraît signée par Leland). La rupture entre eux est consommée par cette collaboration ambiguë, où se mêlent la fidélité spectaculaire de Kane à la vérité (il sait bien que sa femme chante mal) et le faux manifeste: le moment de vérité se solde par la publication d'un mensonge. Cornish, lui, éclaire d'une autre manière cette confusion du vrai et du faux: son tableau, sa seule création, Les Noces de Cana, est admiré et analysé par un public d'experts qui authentifient son appartenance au gothique tardif et finit par occuper «une place d'honneur dans un grand musée des États-unis, regardé par des amoureux de la peinture et d'innombrables étudiants des Beaux-Arts dont les diplômes garantissaient l'infaillibilité de leur savoir et de leur goût ${ }^{9}{ }^{\prime}$. Cette mystification, cette imposture n'enlève rien à la vérité d'un tableau où "il avait assumé son âme ${ }^{10}$ ", qui est «l'allégorie de sa propre vie ${ }^{11}$ ", qui lui a permis de découvrir "(sa) légende, (son) mythe personnel ${ }^{12}$ ». Le roman se conclut sur cette contradiction et évoque pour finir un autre tableau, L'Allégorie de l'amour, de Bronzino:

Dans ce tableau qu'il aimait tant, le Temps et sa fille la Vérité dévoilaient le spectacle de ce qu'était l'amour, tout comme un jour ils dévoileraient Les Noces de Cana. Et quand ce jour viendrait, on parlerait d'abord très durement de duperie et de falsification. Mais Bronzino n'avait-il pas dit beaucoup de choses pertinentes à ce sujet dans son personnage, magnifiquement peint, de la Tromperie, à la fois jeune fille au doux visage qui offre un rayon de miel et scorpion dont la

9. Ibid., p. 512.

10. Ibid.

11. Ibid.

12. Ibid., p. 275. 
partie inférieure est pourvue des griffes du dragon chtonien et de la queue cinglante du serpent ${ }^{13}$ ?

Pour conclure sur ce point, il faudrait rappeler que le dernier film de Welles, réalisé en 1973, en anglais $F$ for Fake, traduit en français par Vérités et mensonges, développait une réflexion sur le vrai et le faux à partir de plusieurs exemples de faussaires.

Les objets ambigus que sont les vies fictives de Kane et de Cornish nous sont présentés indirectement, sous le regard d'un enquêteur. D'emblée, le travail du biographe est donné comme contexte de l'histoire racontée, qui affectera profondément la forme du récit: juste après la séquence d'ouverture, consacrée au dernier soupir de Kane, le film de Welles insère un assez long pastiche des actualités de l'époque, "News on the March", que Dominique Château commente ainsi :

Les détails véridiques, concernant l'histoire des États-unis ou la vie de Hearst, et les faits inventés s'étayent les uns les autres dans un tissu serré qui veut nous faire accroire qu'il a valeur de biographie. [...] Maquillée mais latente au niveau élémentaire, la fiction redevient manifeste dès lors que «l'Actualité en marche» entre en rapport avec le reste du film. Or, les faits donnés pour actuels dans cette portion et les faits donnés pour fictifs dans le reste se recoupent, se chevauchent ou se complètent. [...] S'opère un entrecroisement indécidable entre les deux modes de conditionnement filmique (actualité/fiction) et les deux catégories de faits (empruntés/inventés) ${ }^{14}$.

Cette nécrologie «indécidable» est aussitôt disqualifiée, c'est un brouillon qui suscite un débat dans la salle de rédaction où il vient d'être projeté: «Ce qui lui manque, c'est un angle valable [...] Indispensable, cet angle. [...] Pas de doute, il nous faut un angle." Tout en ironisant sur le jargon et les pratiques journalistiques, Welles fait sienne cette nécessité, comme le démontre, non pas tant l'acharnement du reporter-détective, qui sera désormais le fil conducteur entre les récits successifs des principaux témoins de la vie de Kane, à justifier «l'angle» retenu ("Rosebud»! Qu'est-ce que ça peut signifier?»), mais le dispositif narratif choisi, cette fois, par le cinéaste, qui consiste à porter sur le même objet (Kane) des éclairages divergents, différents "angles de vue». Le film met donc à l'épreuve conjointement deux genres à un moment donné de leur histoire: le genre biographique tel que les supports populaires d'information de l'époque le pratiquent, avec didactisme et indiscrétion; et la fiction, engagée depuis James dans un processus de rénovation qui suppose une déconstruction de la narration traditionnelle

13. Ibid., p. 513.

14. D. Château, Citizen Kane, op. cit., p. 21-23. 
et la multiplication des points de vue, processus qui concerne aussi bien le cinéma que la littérature (on a d'ailleurs comparé Citizen Kane à Rashomon de Kurosawa, tourné dans la même décennie).

Le roman de Davies commence, quant à lui, par mettre en scène trois personnages dont l'un, Simon Darcourt, s'est proposé d'écrire la biographie de Cornish et s'ouvre à ses amis des difficultés qu'il rencontre. Ce prologue, nettement distinct du reste (Simon Darcourt ni ses comparses ne réapparaîtront par la suite), et au cours duquel Simon lit à haute voix la notice biographique publiée par le Times à la mort de Cornish, s'interroge sur la possibilité même de l'écriture du roman qu'il introduit. La première phrase est provocatrice: "Il faut abandonner ce projet de livre ${ }^{15}$ ». Elle est vite nuancée par cette question: «L'esprit de Francis Cornish s'oppose-t-il à ce qu'on écrive sa biographie ${ }^{16}$ ? "Suit la transcription de l'article du Times qui, comme "L'Actualité en marche» dans Citizen Kane, donne au lecteur les principales informations factuelles, et ainsi authentifiées, relatives à la vie du sujet. Mais, là encore, le pseudo compte rendu journalistique se révèle décevant, inapte à restituer la vérité du personnage. Simon conclut donc ainsi :

Évidemment, je pourrais inventer. Si seulement j'osais le faire, si seulement j'avais l'indécence d'un si grand nombre de biographes! Je ne vous parle pas de grossiers mensonges, mais d'une sorte d'affabulation qui peut atteindre à l'art et, d'une certaine manière, correspondre à la vérité. Rappelez-vous ce qu'a dit Browning: «L'art reste le seul moyen possible de dire la vérité, du moins pour des voix comme la mienne». Je servirais Francis tellement mieux si j'avais la liberté d'expression d'un romancier ${ }^{17}$.

Plus loin, Davies attribue une opinion identique à un personnage de faussaire, initiateur de Cornish, qui dit ceci: "L'art est une façon de dire la vérité ${ }^{18}$ ", et cite une formule de Matisse: "L'exactitude, ce n'est pas la vérité $^{19}$ ", qui fait d'ailleurs écho à la conclusion de $F$ for Fake: "L'art est un mensonge qui fait comprendre la réalité. » C'est logiquement dans cette voie que s'engage formellement le roman, c'est-à-dire dans celle de la fantaisie: en effet, la défection de Simon est relayée, compensée, par l'apparition de deux compères, qui regardent défiler la vie de Francis Cornish : l'un est son ange gardien, le petit Zadkiel, qui occupe officiellement, dans la hiérarchie céleste, la fonction de biographe; son interlocuteur est le daimôn de Francis,

15. R. Davies, Un homme remarquable, op. cit., p. 11.

16. Ibid., p. 13.

17. Ibid., p. $27-28$

18. Ibid., p. 391

19. Ibid., p. 399. 
Maimas, son démon au sens mythologique du terme, génie plus protecteur que malfaisant. Leur dialogue scande régulièrement la progression du récit, l'interrompt, la commente, un récit qui par ailleurs obéit conventionnellement à la forme biographique.

Chez Davies, comme chez Welles, la fiction biographique met en cause la biographie et la fiction: Cornish commence sa collection de livres d'art en achetant un exemplaire d'occasion des Vies des plus excellents peintres, sculp teurs et architectes de Vasari, qui devient sa lecture de chevet, mais il remarque, au moment de mourir, que si l'identité du Maître Alchimique, auteur supposé des Noces de Cana, venait à être découverte, "on écrirait des vies du Maître Alchimique, mais celles-ci approcheraient-elles jamais de la vérité, voire des faits ${ }^{20}$ ?».

Dans les deux cas, c'est donc la fiction seule qui permet d'approcher cette vérité - ce qui, en tout cas, passe pour la vérité - et qui le permet au lecteur ou au spectateur seulement: l'avant-dernier plan de Citizen Kane réserve au spectateur la révélation de l'énigme "Rosebud», après que le journaliste a quitté Xanadu ; chez Davies, le sens caché du tableau allégorique de Cornish, Les Noces de Cana, n'est accessible qu'au lecteur et renvoie, comme la luge de Kane, à l'enfance du personnage, relevant d'une conception freudienne du psychisme. Il reste théoriquement caché à Simon Darcourt, à moins de supposer (ce que le roman ne confirme ni ne dément) qu'il est l'auteur du livre, que ce que nous lisons est le résultat de la conversation préliminaire au cours de laquelle il regrettait les pouvoirs du romancier, le résultat aussi du whisky qu'il a bu ce soir-là et des rêves qu'il aura faits. Ce jeu avec le récepteur est fondé sur un postulat confortable, un contrat préalable, qui donne à l'enquête biographique une tonalité policière: il y aurait dans toute vie, dans toute identité, un élément fondamental, une clef, un sens unique. Les deux œuvres satisfont cette promesse: le sens de «Rosebud» et «ce qui a été mis dans la moelle» sont finalement révélés.

Mais les œuvres elles-mêmes démentent cette élucidation réductrice: Kane et Cornish sont partout caractérisés par leur duplicité, leur multiplicité. Le premier est à la fois décrit comme communiste et fasciste, pacifiste et belliciste, capitaliste et anarchiste, et il est encadré par les figures de Bernstein, le Juif, et de Leland, le Puritain. Le dernier plan du film dans lequel il apparaît est explicite: après que Susan l'a quitté, il saccage tout dans

20. Ibid., p. 512. 
sa chambre à l'exception de la petite boule de verre enneigée, prononce le mot magique, "Rosebud», puis traverse le hall sous les yeux de tout son personnel et passe entre deux miroirs qui réfléchissent ainsi sa silhouette à l'infini. C'est sur cette vision démultipliée que Kane, prétendument monolithique, quitte la scène. Cornish, objet du double regard de Zadkiel et Maimas, est canadien et anglais, catholique et protestant, mercurien et saturnien, masculin et féminin, essentiellement ambivalent.

L'équivoque ne se limite pas à l'identité des héros, elle contamine évidemment le récit, qui reproduit formellement leur ambiguïté et interdit toute interprétation exclusive. Dans Citizen Kane, c'est le journaliste, l'investigateur, qui nous le dit. À peine esquissée, son ombre apparait presque toujours de dos, en amorce; les verres de ses lunettes seuls accrochent la lumière, réduisant le personnage à sa fonction et l'identifiant au spectateur, et ses derniers commentaires sont ceux-ci : "Aucun mot ne peut suffire à expliquer la vie d'un homme. Non, pour moi, "Rosebud» ça n'est qu'un morceau du puzzle... une pièce manquante.» Il est possible d'en dire autant de la solution dont cet œil est privé: comme le rappelle Dominique Château,

on a souvent discuté pour savoir si le film de Welles répondait à l'énigme qu'il posait ou bien s'il la laissait pendante. La déception des téléspectateurs devant le film amputé montre au moins que l'absence de la fin laisse le récepteur dans un état de frustration ${ }^{21}$. Mais on semble également fondé à objecter que cette révélation en dernière instance ne fait porter sur l'énigme qu'un éclairage partiel. Le «No Trespassing" (défense d'entrer) qui inaugure le film entier, et que l'on revoit tout à la fin, signifierait-il effectivement, comme le dit, par exemple, Jean Mitry, "qu'on ne peut pénétrer à l'intérieur d'une conscience» 22 ?

Ou encore, que la révélation du secret n'épuise pas les significations du film. L'échec de la transgression biographique ou critique, manifesté par le retour de cette interdiction, "No Trespassing", est décelable rétrospectivement dans l'ambiguïté de la scène d'ouverture: ce n'est qu'après le dernier mot de Kane ("Rosebud»), que l'infirmière ouvre la porte et pénètre dans la pièce, et pourtant ce mot est su, répété, publié, devient le moteur d'une enquête. De même, nous savons tous ce qu'il signifie, et pourtant nous continuons d'explorer, de questionner le film.

Le statut réflexif du secret est encore plus net chez Davies, qui choisit de faire de son héros un peintre, qui s'exerce au portrait, débute précisément

21. La diffusion à la télévision américaine d'une version de Citizen Kane tronquée pour obéir au rythme imposé des coupures publicitaires et privée du dernier plan aurait déclenché l'indignation des téléspectateurs.

22. D. Château, Citizen Kane, op. cit, p. 60. 
comme caricaturiste et dont les premiers modèles - involontaires - sont des cadavres, observés et croqués dans une morgue. À l'instar du biographe, il tente de redonner vie à leurs traits et de nombreux propos tenus dans le roman au sujet de la peinture pourraient s'appliquer à la fiction biographique telle que la pratiquent Davies, et, ce qui est plus étonnant, Orson Welles: «Un portrait exprime un jugement de l'artiste tout autant qu'il offre une image ressemblante ${ }^{23}$ "; " un tableau est plusieurs choses: ce que voit l'artiste, mais aussi ce qu'il pense de ce qu'il voit, et, de ce fait, c'est dans une

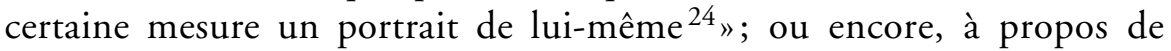
Cornish, "c'est un homme qui adore peindre sous forme de devinettes et de clins d'œil au spectateur ${ }^{25}$ ". Les spéculations des experts sur le sens des Noces de Cana ne sont pas disqualifiées en elles-mêmes mais pour leur prétention à réduire la vérité de l'œuvre à un "code», qu'il soit alchimique ou politicoreligieux. "Personne ne connaît jamais tous les aspects d'une histoire ${ }^{26}$ ", commente le narrateur quelques lignes avant la fin, ouvrant l'œuvre à toutes les lectures, y compris à celle, internationale, esquissée pour commencer: estce un hasard si Cornish décide d'exprimer sa «légende», son «mythe personnel» en peignant Les Noces de Cana? ou est-ce, entre autres, par association avec le nom de son pays, "Cana»/da, de Kanata, qui voudrait dire, en Iroquois: «Il n'y a rien là»? Le tableau, dans cette perspective, dirait l'ambition fondatrice du roman, la nécessité d'inventer la fiction du Nouveau Continent.

23. R. Davies, Un homme remarquable, op. cit, p. 110.

24. Ibid., p. 286.

25. Ibid., p. 477.

26. Ibid., p. 510 . 